\title{
Parallel communications for ATM network control and management ${ }^{\star}$
}

\author{
Tony T. Lee*, Soung C. Liew ${ }^{1}$, Quan-long Ding ${ }^{2}$ \\ Department of Information Engineering, The Chinese University of Hong Kong, Shatin, New Territories, Hong Kong
}

Received 26 June 1995; revised 9 September 1996

\begin{abstract}
This paper describes an end-to-end parallel communications scheme based on a vector routing algorithm (VRA) for ATM network control and management. An information string is partitioned into $m$ parts, which are then coded into $k>m$ parts and sent out on $k$ separate subchannels to the receiver. When $\dot{m}$ of the $k$ parts are received correctly, the original information can be reconstructed. Two desirable effects are achieved in the context of ATM traffic control: (1) the burstiness of the source traffic can be smoothed out by the partition process; (2) the quality of service in terms of error, loss and delay can be controlled using the number of redundant routes $k-m$ as a control parameter. Our results show that VRA is especially suitable for services with highly bursty traffic. We argue that several network management issues, including reliability, evolution and integration, security, and administration and billing can be addressed in a simple manner using the VRA framework. (C) 1997 Published by Elsevier Science B.V.
\end{abstract}

Keywords: ATM; Parallel communications; Network control and management; Diversity routing; Quality of service

\section{Introduction and overview}

The Asynchronous Transfer Mode (ATM) has been accepted by CCITT as the multiplexing and switching technique for the future Broadband Integrated Services Digital Network (B-ISDN). In an ATM network, messages are divided into fixed length packets called cells. Cells are routed in the network based on the destination information contained in their header. ATM is envisioned as a platform for supporting bandwidth-on-demand services, and the transport of all services is expected to use the same cell format.

An ideal connection in an ATM network should preserve the following quality-of-service (QOS) attributes:

QOS 1. The content of information: The information should not be distorted.

\footnotetext{
^ Supported by a Hong Kong UGC Earmarked Grant: CUHK 269/94E.

* Corresponding author. Tel.: 852 26098355; fax: 852 26035032; e-mail: ttlee@ ie.cuhk.edu.hk.

${ }^{1}$ E-mail: soung@ie.cuhk.edu.hk.

${ }^{2}$ E-mail: qlding3@ie.cuhk.edu.hk.
} 
QOS 2. Information should not be misrouted or lost and should be received in the same order as it was generated by the sender.

QOS 3. The statistics of the packet stream: If the packets were generated at time epochs $t_{1}, t_{2}, t_{3}, \ldots$, then they should be received at time $t_{1}+T, t_{2}+T, t_{3}+T, \ldots$, where $T$ is a constant delay.

Unfortunately, perfect connections do not exist in real networks, due to competition among cells from different connections for the same network resources. Despite many resource allocation and congestion control mechanisms proposed recently, it is still difficult to guarantee the quality of service for each individual connection while maintaining efficient bandwidth usage of the network resources, especially when the traffic is highly bursty in nature.

This paper proposes an end-to-end parallel communications scheme, the vector routing algorithm (VRA), that achieves QOS guarantees in an efficient manner. It decomposes a channel into a number of subchannels. Traffic from the source is partitioned into streams consisting of $m$ packets each. The $m$ packets in each information stream is then encoded into $k>m$ packets, which are then sent along $k$ separate routes in parallel. The original information can be reconstructed by the receiver if any $m$ of these $k$ packets are received correctly in a timely manner. The quality of service, as measured by the delay jitter and packet loss, is controlled by the redundancy $k-m$.

There are three levels of route diversity appropriate for the implementation of VRA in a network. First, the separate subchannels of VRA can be implemented on different physical routes. Alternatively, the subchannels can be implemented along the same route, but separate physical links between two nodes in the route will be used (e.g., different fibers of a fiber bundle). Finally, the same physical link may consist of several subchannels, whose data streams do not intermix in the multiplexing process. VRA also works even if the paths of some subchannels overlap, although the advantage will be reduced.

VRA can be implemented in the adaptation layer at the edge of the ATM network (i.e., at the sender's and receiver's ends) so that "imperfections" introduced by the transport process of the network can be removed before the data are forwarded to the receiving user. The splitting of a main channel into $k$ subchannels will then occur in this layer. The ATM adaptation layer (AAL) supports a variety of services, which can be classified broadly into four classes:

(1) Class A: Circuit emulation services.

(2) Class B: Variable bit rate services with time synchronization between sender and receiver(s).

(3) Class C: Connection oriented data services.

(4) Class D: Connectionless data services.

However, because of the drastically different characteristics of the traffic in different service class, it is generally very difficult to control the congestion level inside the network. For example, cells could be discarded inside the network because of bursty traffic arrivals at a switching node. Cells successfully received at the receiver may have experienced large delay variance. This paper will discuss how VRA can be used to transport these four classes of traffic.

A very important property of VRA in the context of ATM networks is that the sequence order of reception between the $k$ packets in the same batch is immaterial as far as decoding is concerned; only the ordering between successive batches must be preserved, and this is guaranteed if each subchannel maintains the sequence order of cells. In addition, since any $m$ of the $k$ packets will be sufficient for decoding, the $k$ subchannels can be selected and treated independently even though their routes from the source to destination may have some common links, multiplexers or switch nodes.

Ref. [3] describes a parallel routing concept developed within the European RACE project ATMOSPHERIS-R1014 called "string mode". The main idea is to decompose highly bursty traffic from 
a source to a number of smaller smoother traffic strings. Lacking is the concept of using redundancy in the form of information coding to guarantee QOS. Parallel routing schemes with coding have also been proposed previously $[7,11]$ to achieve hetter fault tolerance and delay performance. Our study focuses on using the framework as a unified framework to control the QOS in ATM networks: how parameters $m$ and $k$ can be regulated to achieve QOS objectives. In addition, a recursive decoding algorithm is proposed to reduce the time lag between the arrival of the $m$ th packet and the completion of the decoding process, making VRA more attractive for real-time services.

The remainder of the paper is organized as follows. Section 2 discusses the basics of VRA and provides a recursive decoding algorithm. Reduction of traffic burstiness is shown quantitatively. Section 3 establishes several fundamental results showing that the QOS can be controlled using the VRA parameters $m$ and $k$. Section 4 describes the performance evaluations by simulation. Section 5 describes control and management issues in ATM networks. Finally, Section 6 concludes by providing further research directions based on this work.

\section{Parallel communications}

A communications channel for broadband services can be parallelized by the VRA described in this section. The basic concept of VRA is derived from the Reed-Solomon code [12]. We briefly describe the basics of channel decomposition and reconstruction before going into the details in the subsections. A string of information $I=\left(b_{0}, b_{1}, \ldots, b_{m-1}\right)$ can be represented by a polynomial

$$
f(x)=b_{m-1} x^{m-1}+\cdots+b_{1} x+b_{0}
$$

over some finite field $G F(q)$. Substituting for $x$ with $n(m \leq n<q)$ distinct elements $a_{0}, a_{1}, \ldots, a_{n-1} \in$ $G F(q)$ yields $n$ values for $f(x), C=\left(f\left(a_{0}\right), f\left(a_{1}\right), \ldots, f\left(a_{n-1}\right)\right)$.

Suppose we send any subset of $k, m \leq k \leq n$, elements of $C$ from the source node to the destination node along $k$ different routes. When the destination node receives any $m$ elements out of these $k$ elements correctly, the original string of information $I$ is reconstructed using polynomial interpolation. That is, suppose the destination knows $a_{i}$ a priori. Upon receiving $m$ elements of $C$, it forms $m$ linearly independent equations from (1), the solution of which yields $I=\left(b_{0}, b_{1}, \ldots, b_{m-1}\right)$. This scheme disperses the burst of information $I$ along $k$ parallel routes such that each route carries only $|I| / m$ amount of information.

We observe the following similarities between the above decomposition and reconstruction processes and the Nyquist's sampling theorem [10]:

(1) Decompose the original information by taking "samples".

(2) Require minimal number of samples to reconstruct the original information.

(3) Reconstruct the original information from samples by interpolation, a linear transformation. The investigation of VRA in the context of ATM networks is discussed in the following sections.

\subsection{Vector routing algorithm}

Let $K=G F\left(2^{s}\right)$ be the vector space over $Z_{2}$ with basis $1, \alpha, \alpha^{2}, \ldots, \alpha^{s-1}$, where $\alpha$ is the root of some irreducible polynornial of degree $s$ over $Z_{2}$. The non-zero elements of $K$ form a multiplicative cyclic group of order $2^{s}-1$. Suppose a string of information

$$
I=\left(b_{1,1}, \ldots, b_{1, m}, b_{2,1}, \ldots, b_{2, m}, \ldots, b_{l, 1}, \ldots, b_{l, m}\right)
$$



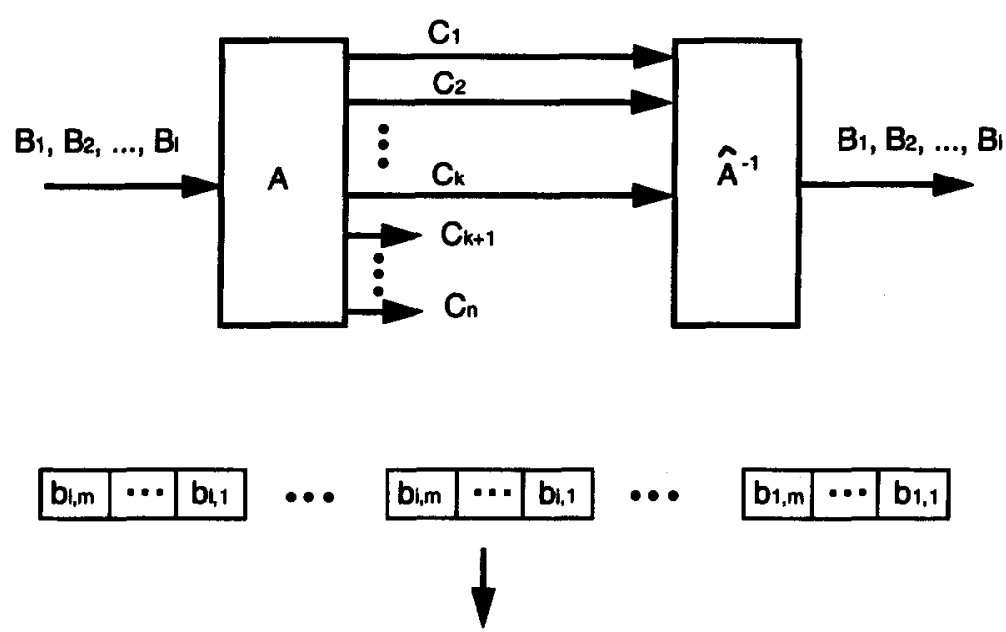

$c_{1}$

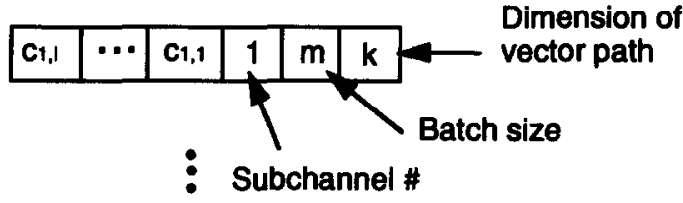

$C_{k}$

\begin{tabular}{|l|l|l|l|l|l|}
\hline$c_{k, 1}$ & $\cdots$ & $c_{k, 1}$ & $k$ & $m$ & $k$ \\
\hline
\end{tabular}

I: packet payload

m: batch size

$\mathrm{k}$ : dimension of vector paths

Fig. 1. Basic scheme of vector routing.

is transmitted from a source to a destination, where cach $b_{i, j} \in K$ is an $s$-bit symbol. With reference to Fig. 1, this string of information is partitioned into sequences of length $m$, represented in vector form as

$$
B=\left[\begin{array}{c}
B_{1} \\
\vdots \\
B_{j} \\
\vdots \\
B_{l}
\end{array}\right]=\left[\begin{array}{cccc}
b_{1,1} & b_{1,2} & \cdots & b_{1, m} \\
\vdots & & & \vdots \\
b_{j, 1} & b_{j, 2} & \ldots & b_{j, m} \\
\vdots & & & \vdots \\
b_{l, 1} & b_{l, 2} & \ldots & b_{l, m}
\end{array}\right]
$$

This sequence of information will be encoded into a sequence of $n$ code words by matrix transformation. Let 


$$
A=\left[\begin{array}{c}
A_{1} \\
\vdots \\
A_{i} \\
\vdots \\
A_{n}
\end{array}\right]=\left[\begin{array}{cccc}
a_{1,1} & a_{1,2} & \cdots & a_{1, m} \\
\vdots & & & \vdots \\
a_{i, 1} & a_{i, 2} & \cdots & a_{i, m} \\
\vdots & & & \vdots \\
a_{n, 1} & a_{n, 2} & \cdots & a_{n, m}
\end{array}\right]
$$

be an $n \times m$ matrix, where each vector $A_{i} \in K^{m}$ and every $m$ different vectors of the set $\left\{A_{1}, \ldots, A_{n}\right\}$ are linearly independent. The choice of the matrix is arbitrary. One way is to choose $n$ different elements $\alpha_{1}, \ldots, \alpha_{n} \in K$ and set

$$
A_{i}=\left[\begin{array}{lllll}
1 & \alpha_{i} & \alpha_{i}^{2} & \cdots & \alpha_{i}^{m-1}
\end{array}\right], \quad 1 \leq i \leq n .
$$

Then, any $m$ of these vectors are linearly independent, since the matrix consisting of these vectors is of the form of the Vanderrnonde matrix, whose determinant is given by

$$
\left|\begin{array}{ccccc}
1 & \alpha_{(1)} & \alpha_{(1)}^{2} & \cdots & \alpha_{(1)}^{m-1} \\
1 & \alpha_{(2)} & \alpha_{(2)}^{2} & \cdots & \alpha_{(2)}^{m-1} \\
\vdots & & \vdots & & \vdots \\
1 & \alpha_{(m)} & \alpha_{(m)}^{2} & \cdots & \alpha_{(m)}^{m-1}
\end{array}\right|=\prod_{i<j}\left(\alpha_{(i)} \oplus \alpha_{(j)}\right) \neq 0
$$

The $n$ coded words can be obtained simply by the matrix multiplication

$$
C=\left[\begin{array}{c}
C_{1} \\
C_{2} \\
\vdots \\
C_{n}
\end{array}\right]=A B^{\mathrm{T}}=\left[\begin{array}{c}
A_{1} \\
A_{2} \\
\vdots \\
A_{n}
\end{array}\right]\left[\begin{array}{llll}
B_{1}^{\mathrm{T}} & B_{2}^{\mathrm{T}} & \cdots & B_{l}^{\mathrm{T}}
\end{array}\right]=\left[\begin{array}{cccc}
A_{1} B_{1}^{\mathrm{T}} & A_{1} B_{2}^{\mathrm{T}} & \cdots & A_{1} B_{l}^{\mathrm{T}} \\
A_{2} B_{1}^{\mathrm{T}} & A_{2} B_{2}^{\mathrm{T}} & \cdots & A_{2} B_{l}^{\mathrm{T}} \\
\vdots & & & \vdots \\
A_{n} B_{1}^{\mathrm{T}} & A_{n} B_{2}^{\mathrm{T}} & \cdots & A_{n} B_{l}^{\mathrm{T}}
\end{array}\right] .
$$

Suppose the dimension of vector path $k$ ( $\left.m \leq k \leq n \leq 2^{s}-1\right)$ coded words are transmitted through $k$ different routes, and the first $m$ of them received are $C_{(1)}, C_{(2)}, \ldots, C_{(m)}$. Since

$$
\hat{C}=\left[\begin{array}{c}
C_{(1)} \\
C_{(2)} \\
\vdots \\
C_{(m)}
\end{array}\right]=\hat{A} B^{\mathrm{T}}=\left[\begin{array}{c}
A_{(1)} \\
A_{(2)} \\
\vdots \\
A_{(m)}
\end{array}\right]\left[\begin{array}{llll}
B_{1}^{\mathrm{T}} & B_{2}^{\mathrm{T}} & \ldots & B_{l}^{\mathrm{T}}
\end{array}\right]
$$

and $\hat{A}$ is non-singular, the receiver can obtain the original information by the decoding process $B=$ $\left(\hat{A}^{-1} \hat{C}\right)^{\mathrm{T}}$. Note that the reception order of the packets is immaterial as far as the decoding process is concerned.

For ATM applications, if we choose $s=8$, then each $b_{i, j}$ is an 8-bit byte. If each code word $C_{i}$ is carried on the paylcad of an ATM cell, the code word length $l=48$ bytes. Each connection can have up to $2^{s}-1=255$ subchannels. Each information string $I$ contains $m$ packets. Thus, the parameter $m$ is referred to as the batch size. A burst of information arrivals may be encoded into a number of batches, each with $m$ packets. If $m$ is larger than the burst length, then empty bytes will be encoded, resulting in some overhead. Thus, it is important to choose an $m$ appropriately to match the burst length. 


\subsection{Recursive algorithm}

The recursive algorithm for the encoding process is obvious: as $B_{i}$ arrives at the encoder, a column of $C$ can be calculated by the multiplication $A B_{i}$.

It is desirable to have a recursive computation scheme at the receiver, especially for real-time traffic. The following recursive algorithm allows computation of partial results for $\hat{A}^{-1}$ each time a new packet is received from a subchannel, thus fully exploiting the waiting time for the $m$ packets to arrive.

Let us denote an $i \times i$ submatrix of $\hat{A}$ by $\hat{A}(i)$. We can write

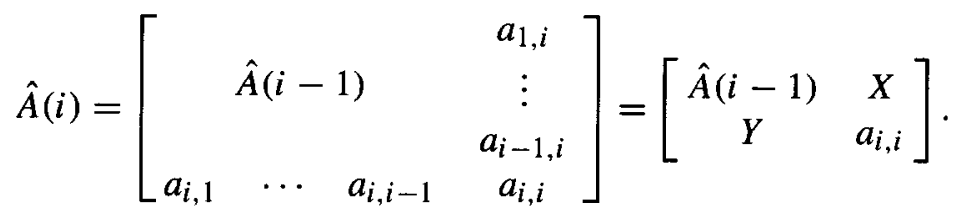

It can be easily verified that

$$
\hat{A}^{-1}(i)=\left[\begin{array}{cc}
\hat{A}^{-1}(i-1)+d^{-1} \hat{A}^{-1}(i-1) X Y \hat{A}^{-1}(i-1) & -d^{-1} \hat{A}^{-1}(i-1) X \\
-d^{-1} Y \hat{A}^{-1}(i-1) & d^{-1}
\end{array}\right],
$$

where $d=a_{i, i}-Y \hat{A}^{-1}(i-1) X$ is Schur's complement of $\hat{A}(i)$ with respect to $\hat{A}(i-1)$. Retrieving $X, Y$ and $a_{i, i}$ as soon as the $i$ th packet is received, we see that $\hat{A}^{-1}(i)$ can be obtained from previously computed $\hat{A}^{-1}(i-1)$. After the computation of $\hat{A}^{-1}(m), B^{\mathrm{T}}$ is readily calculated from $\hat{A}^{-1}(m) \hat{C}(m)$, where $\hat{C}(m)=\left[\begin{array}{llll}C_{(1)} & C_{(2)} & \cdots & C_{(m)}\end{array}\right]^{\mathrm{T}}$.

\subsection{Controllability of traffic burstiness}

In this section, we investigate the smoothing effect of VRA on bursty source traffic. To simplify our discussion, we model the burstiness of the traffic stream in a similar manner as the noise in electronic circuits. Thus, manipulations used in the calculation of noise can be readily adopted here. The ways in which burstiness can be easily calculated motivates our formulation.

Consider the traffic arrival process of a connection at the source. Let us assume that time is slotted and that packets may arrive only at the beginning of each slot and multiple packets may arrive simultaneously in a group. We also assume for the time being that the group size is a multiple of $m$; results without this assumption will be presented after the following derivation. Denote the number of packets arriving in time slot $i$ by

$$
y[i]=m f[i]
$$

where $f[i]$ is an integer random variable. We assume $y[i]$ is stationary with expectation $E[y[i]]=\rho$. Traffic burstiness will be defined in terms of the deviation from the expected arrival rate $\rho$ over an observation time window $T$. Let

$$
Y[i]=\frac{1}{T} \sum_{i=T<i} y[j]
$$




$$
N[i]=\frac{1}{T}\left[\sum_{i-T<j \leq i} y[j]-\rho T\right]=Y[i]-\rho .
$$

Note that $\sum_{i-T<j \leq i} y[j]$ is the actual number of arrivals and $\rho T$ is the expected number of arrivals over the past $T$ slots. Thus, $N[i]$ is the deviation from the expected arrivals normalized by $T$. Physically, $T$ is a "time constant" related to the system under study. For example, the $T$ for a multiplexer with only a few buffers should be set small, since it is unlikely that arrivals long ago will significantly affect the current system behavior.

The deviation $N[i]$ can be negative. To obtain a positive quantity, burstiness at time $i$ is defined as

$$
B[i]=E\left[N^{2}[i]\right]
$$

Consider a subchannel $p$ resulting from vector routing. With the assumption of multiple- $m$ burst length, its arrival process is given by

$$
y_{p}[i]=\frac{y[i]}{m}==f[i]
$$

with $E\left[y_{p}[i]\right]=\rho / m$. The deviation at time $i$ is $N_{p}[i]=N[i] / m$. Thus, the burstiness is

$$
B_{p}[i]=\frac{B[i]}{m^{2}}
$$

We see that the burstiness has been reduced by $m^{2}$ times.

For a fair comparison, let us assume that there are $m$ connections. As shown in Fig. 2, information from the $m$ connections is dispersed and multiplexed over $m k$ subchannels. A composite channel consists of $m$ subchannels from $m$ different connections. Thus, each connection contributes a load of $\rho / m$ to each subchannel so that the load at each composite channel is $\rho$, the same as when there is no vector routing. If

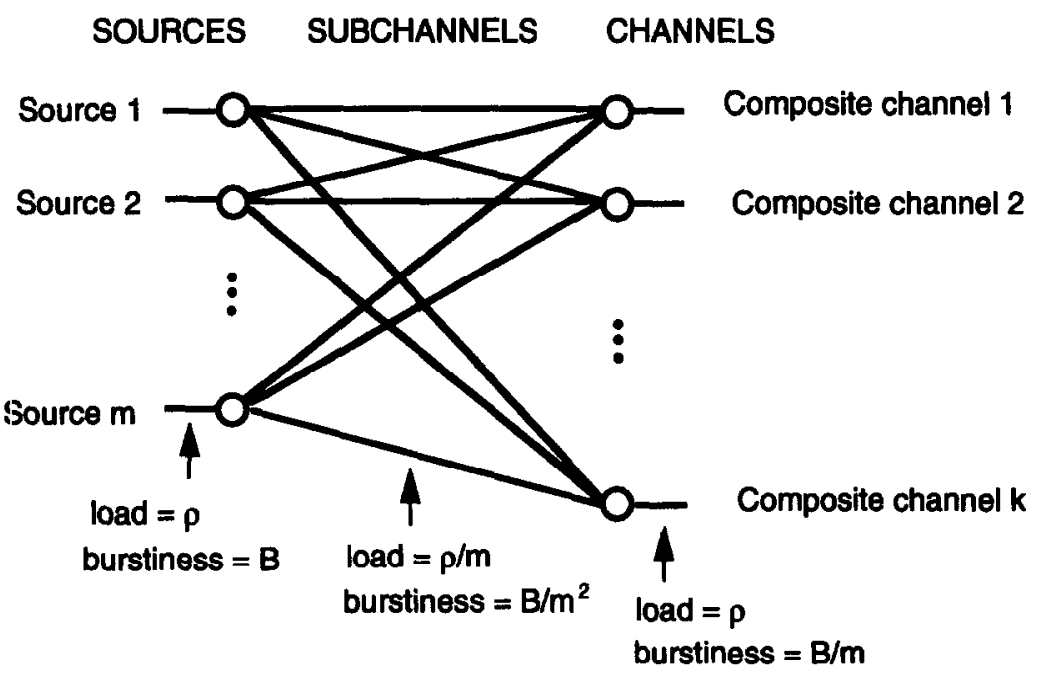

Fig. 2. Dispersal and multiplexing of traffic from $m$ connections to $K$ channels. 
the arrival processes of the $m$ connections are independent, then the burstiness on each composite channel $s$ is given by

$$
B_{s}[i]=m B_{p}[i]=\frac{B[i]}{m}
$$

since the variance of a sum of independent random variables is the sum of the variance of the random variables; this is analogous to adding the variances of independent noise. Note that there is still an improvement factor of $1 / \mathrm{m}$. This result is general and is independent of the distribution of $f[i]$. Furthermore, $f[i]$ for different $i$ need not be independent.

To remove the multiple- $m$ burst-length assumption, we can write

$$
y[i]=m f[i]+g[i],
$$

where $0 \leq g[i]<m, f[i]$ and $g[i]$ not necessarily independent. There are two strategies to the residual packets: (1) Round-up strategy: add $m-g[i]$ dummy packets to force the group size to be a multiple of $m$; (2) No-rounding strategy: disperse the $g[i]$ residual packets to $k^{\prime}\left(g[i]<k^{\prime}<k\right)$ subchannels. The first strategy results in some additional overhead. For the second strategy, if we set $k^{\prime} / g[i] \approx k / m$, there is little additional overhead. Based on the second strategy and with the assumption that $k^{\prime}$ random channels of the $k$ channels are chosen to send the $k^{\prime}$ packets, it is shown in Appendix A that

$$
B_{s}[i]=\frac{B[i]}{m}+\frac{1}{T}\left(E[g[i]]-\frac{E\left[g^{2}[i]\right]}{m}\right) .
$$

Except for cases where the original source traffic is already very smooth (e.g., when $y[i]=1.5 m$ for all $i$ ), the additional term $\left(E[g[i]]-E\left[g^{2}\right] / m\right) / T$ is typically much smaller than $B[i]$ so that the improvement is still quite substantial.

\section{Controllability of quality-of-service (QOS)}

This section shows that the QOS, in terms of robustness against packet errors, packet loss, and packet delay jitter, can be guaranteed using the VRA framework. The trade-offs and inter-relationships between redundancy and QOS will be discussed in detail. Specifically, the delay jitter and reconstruction failure due to packet loss or errors can be made arbitrarily small by increasing $k$; this remains true even if we fix the efficiency factor $m / k$.

Section 3.1 discusses the forward error correction capability of VRA. Although VRA has the error correction capability, our main purpose here is to use it to control the overall quality of end-to-end connections, including load balancing, traffic burstiness, and packet loss and delay. Sections 3.2 and 3.3 address issues related to packet loss and delay.

\subsection{Controllability of information corruption}

VRA can be used to detect and correct errors introduced by the channel noise. A packet is said to be in error if any bit in it is corrupted. Assuming all $k$ packets in a batch are received, it can be shown that VRA is able to correct up to $\frac{1}{2}(k-m-1)$ errors if $k-m$ is odd and $\frac{1}{2}(k-m)$ errors if $k-m$ is even; error detection is possible for up to $\frac{1}{2}(k-m+1)$ errors if $k-m$ is odd and $\frac{1}{2}(k-m)$ errors if $k-m$ is even [12]. 
If additional end-to-end error detection capability is incorporated into each packet. Then, more packet errors can be corrected. Let us suppose that a packet in error can always be detected. Since $m$ correct packets are sufficient for reconstruction, VRA can correct up to $k-m$ error packets, about twice more than without error detection in each packet. The results are summarized below.

QOS 1. With bit-error detection for each packet, VRA is able to correct up to $k-m$ packet errors for each batch of packets. Without bit-error detection for each packet, VRA is able to correct an order of $\frac{1}{2}(k-m)$ packet errors.

It should be pointed out forward error correction has been considered previously $[1,8]$. However, the coded information is sent out in parallel subchannels in our case, the previous studies considered sending the coded information sequentially on the same channel. Sequential transmission is more susceptible to burst errors where the packet errors and loss in a batch are positively correlated. Parallel transmission has the advantage of being more tolerant to burst errors on individual subchannels.

\subsection{Controllability of information loss}

At the receiver end, if less than $m$ packets arrive, then decoding fails. There are two possibilities for the failures of packets to arrive:

(1) Packets are lost inside the network (e.g., due to buffer overflow, switch contention [5], etc.).

(2) For real-time traffic, end-to-end packet delays fail to fall within a preset window $D=\left[T_{\min }, T_{\max }\right]$.

Generally, the window size $T_{\max }-T_{\min }$ is set to correspond to the size of the smoothing buffer at the receiver, and $T_{\max }$ is set to maximize the likelihood of packet reception within the window, subject to the constraint that $T_{\max }$ must be bounded by the maximum tolerable delay.

We note that if a packet is lost in the network, it will also not be received within the window $D$. Thus, we shall simply consider all packets that fail to arrive within $D$ as being lost. Accordingly, we denote by $p$ the probability that the packet from a subchannel arrives within $D$. Although we shall assume this probability to be the same for all subchannels in the following discussion for the sake of simplicity, the qualitative results remain the same even if this were not the case.

Consider the loss probability $Q(m, k)$ that less than $m$ out of $k$ packets from the $k$ subchannels are received within the delay window $D=\left[T_{\min }, T_{\max }\right]$. Assuming that arrivals on different subchannels are independent, $Q(m, k)$ is the first $m$ terms of the binomial distribution with parameter $p$ :

$$
Q(m, k)=\sum_{j=0}^{m-1}\left(\begin{array}{l}
k \\
j
\end{array}\right) p^{j}(1-p)^{k-j} .
$$

An approximate formula of $Q(m, k)$ can be derived by the large deviation principle [15]. Let $Y_{i}=0$ if the packet from subchannel $i$ arrives within $D$ and $Y_{i}=1$ otherwise. Thus, $P\left[Y_{i}=0\right]=p$. The total number of lost: packets is $R_{k}=Y_{1}+Y_{2}+\cdots+Y_{k}$. We have $E\left[R_{k}\right]=k(1-p)$. Assuming $Y_{i}$ are identical and independent distributed random variables, by the large deviation principle, the probability $P\left[Y_{1}+Y_{2}+\cdots+Y_{k} \geq k a\right]$ is given by

$$
P\left[R_{k} \geq k a\right]=: \mathrm{e}^{-k \ell(a)+\mathrm{O}(k)},
$$




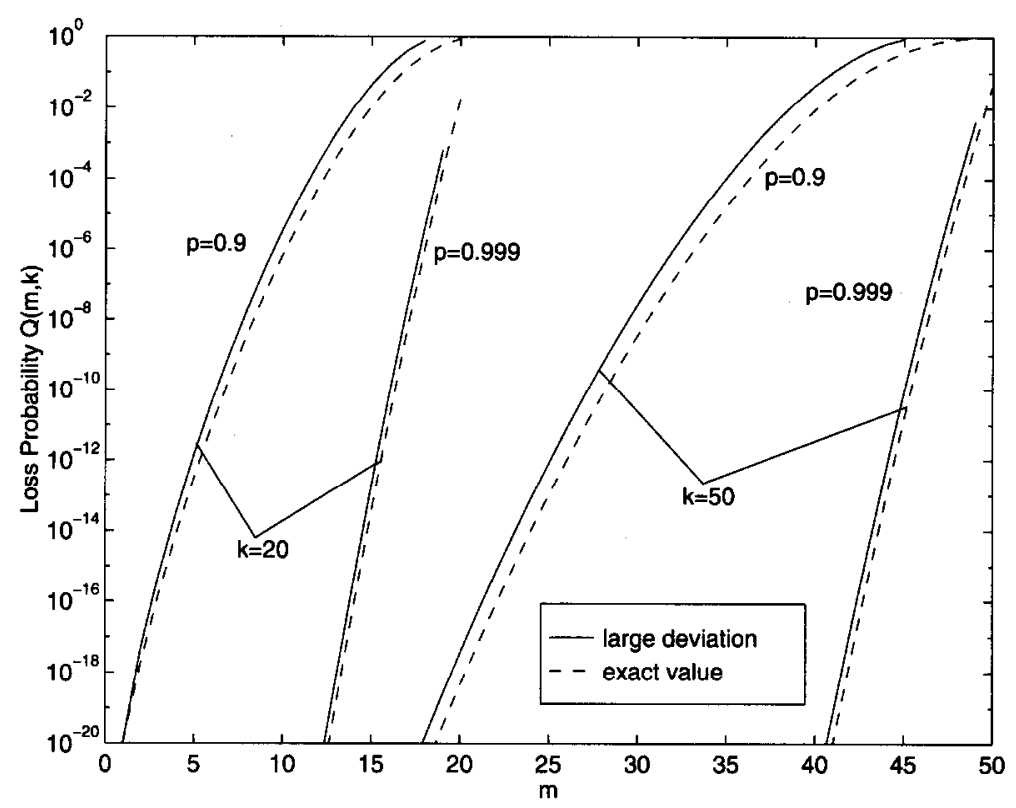

Fig. 3. The loss probability by the large deviation.

where the large deviation rate function $\ell(a)$ is given by

$$
\ell(a)=a \log \frac{a}{1-p}+(1-a) \log \frac{1-a}{p}
$$

and $a>E\left[Y_{1}\right]=1-p$.

Let $a=(k-m+1) / k$, from (21) and (22) the loss probability $Q(m, k)$ is given by

$$
Q(m, k)=P\left[R_{k} \geq k-m+1\right] \approx\left(\frac{k-m+1}{k(1-p)}\right)^{-(k-m+1)}\left(\frac{m-1}{k p}\right)^{-(m-1)},
$$

where $m \leq k p$.

To estimate the accuracy of the large deviation method, a numerical comparison of Eqs. (20) and (23) is presented in Fig. 3. As can be seen, the solid curve from Eq. (23) is matched with dish line obtained from Eq. (20) (the error results from order term $\mathrm{O}(k)$ ). In addition, when the probability $p$ approaches to 1 , the results obtained from the two methods are very close. Therefore, Eq. (23) can be used to estimate the loss probability of parallel communications scheme.

The asymptotic behavior can be studied by standard techniques in probability theory. Let $X_{i}=1$ if the packet from subchannel $i$ arrives within $D$ and $X_{i}=0$ otherwise. Thus, $P\left[X_{i}=1\right]=p$. The total number of arrivals within $D$ is $S_{k}=X_{1}+X_{2}+\cdots+X_{k}$. We have $E\left[S_{k}\right]=k p$ and $\operatorname{Var}\left[S_{k}\right]=k$ $\operatorname{Var}\left[X_{i}\right]=k p(1-p)$.

$$
\begin{aligned}
Q(m, k) & =P\left[S_{k}<m\right] \\
& \leq \frac{\operatorname{Var}^{2}\left[S_{k}\right]}{\operatorname{Var}^{2}\left[S_{k}\right]+\left(E\left[S_{k}\right]-m\right)^{2}} \quad \text { for } E\left[S_{k}\right]-m>0
\end{aligned}
$$




$$
=\frac{p(1-p)}{p(1-p)+k(p-c)^{2}}, \quad c<p,
$$

where inequality (25) is obtained by application of the one-sided Chebychev's inequality [14]. It is easy to see from the above that as $k \rightarrow \infty, Q(m, k) \rightarrow 0$. This property is not so obvious intuitively because the efficiency factor $c==m / k$, hence the redundancy factor $1-c$, is kept constant as $k \rightarrow \infty$. The physical requirement of the stable condition $c<p$ (or $k p>m$ ) is obvious, since it would be unreasonable to expect successful decoding if the expected number of successful arrivals is no more than $m$.

A stronger result can be obtained from the central limit theorem [2]. For large $k,\left(S_{k}-k p\right) / \sqrt{k p(1-p)}$ is normally distributed with zero mean and variance one. Specifically,

$$
\begin{aligned}
Q(m, k) & =P\left[S_{k}<m\right]=P\left[\frac{S_{k}-k p}{\sqrt{k p(1-p)}}<\frac{m-k p}{\sqrt{k p(1-p)}}\right] \\
& =\frac{1}{\sqrt{2} \pi}=\int_{-\infty}^{-\sqrt{k}(p-c) / \sqrt{p(1-p)}} \mathrm{e}^{-x^{2} / 2} \mathrm{~d} x+\frac{A}{\sqrt{k}}
\end{aligned}
$$

where $A$ is a numerical constant depending on $p$ [2]. From the above, we obtain the following property:

QOS 2. For a given fixed

$$
\frac{m}{k}=c, \quad Q(m, k) \rightarrow \frac{1}{\sqrt{2 \pi}} \int_{-\infty}^{-\sqrt{k}(p-c) / \sqrt{p(1-p)}} \mathrm{e}^{-x^{2} / 2} \mathrm{~d} x
$$

for large $k$. Furthermore,

$$
\lim _{k \rightarrow \infty} Q(m, k)= \begin{cases}0 & \text { if } c<p \\ 1 & \text { if } c=p \\ 2 & \text { otherwise }\end{cases}
$$

From the service viewpoint, QOS 2 suggests the suitability of VRA for traffic with large burst lengths (see Section 2). This is because the batch size $m$ can then be made larger, and therefore $k$ can be set to a larger value to lower $Q(m, k)$ without increasing the redundancy factor $1-c$, as illustrated in Fig. 4.

A similar result as QOS 2 can be established for decoding failures due to packet errors discussed in Section 3.2. Namely, if $p$ is interpreted as the probability that a packet is received correctly, $Q(m, k)$ will then be the probability of decoding failure due to packet errors, assuming bit-error detection capability is incorporated into each packet. Without bit-error detection for each packet, the probability of decoding failure will approach to zero asymptotically only if $c<2 p-1$. Since $c>0$ physically, $p>\frac{1}{2}$ in order that this is possible.

\subsection{Controllability of information delay}

Since any $m$ packets from the $k$ subchannels can be used to reconstruct the original information, we shall invoke the order statistics to study the end-to-end delay of the $m$ th packet received. Let $T_{i}$ denote the packet 


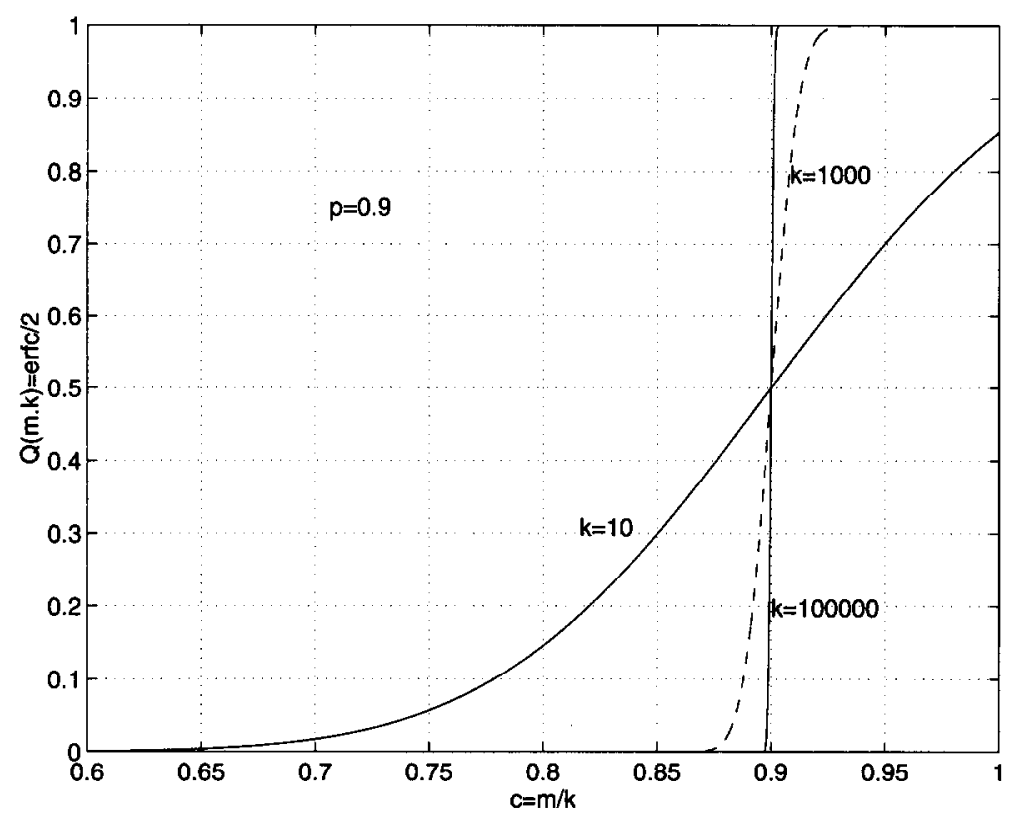

Fig. 4. The loss probability with large $k$ by central limit theorem.

delay on the $i$ th subchannel. We assume $T_{i}$ 's to be independent identically distributed random variables. The delay jitter corresponds to the delay jitter experienced by the $m$ th arriving packet at the receiver.

Let $T_{(i)}$ denote the delay statistic of the $i$ th packet arrived at the receiver. This is the well-known order statistic [13] whose probability density is given by

$$
f_{(m)}(t)=\frac{k !}{(m-1) !(k-m) !} F^{m-1}(t)[1-F(t)]^{k-m} f(t),
$$

where $F(t)$ and $f(t)$ are the probability distribution and density function of $T_{i}$, respectively.

For illustration, consider the case where $T_{i}$ is uniformly distributed between $a$ and $a+b$. The expectation and variance of $T_{(m)}$ are given by

$$
\begin{aligned}
E\left[T_{(m)}\right] & =a+b \frac{m}{k+1}, \\
\operatorname{Var}\left[T_{(m)}\right] & =b^{2} \frac{m}{(k+1)(k+2)}\left[1-\frac{m}{k+1}\right] .
\end{aligned}
$$

If we keep $m$ constant and let $k$ increase, both $E\left[T_{(m)}\right]$ and $\operatorname{Var}\left[T_{(m)}\right]$ decrease, revealing the trade-off between delay jitter and redundancy. This illustrates the trade-off between bandwidth and delay jitter, since much redundancy is needed to reduce $\operatorname{Var}\left[T_{(m)}\right]$ arbitrarily close to zero.

A stronger finding can be obtained from the following result [4] that is somewhat similar to the central limit theorem: Let $m=[k \lambda]+1$, where $[x]$ denote the integer part of $x$, and $\xi_{\lambda}=F^{-1}(\lambda)$. If $0<f\left(\xi_{\lambda}\right)<$ $\infty$, then as $k \rightarrow \infty$, the asymptotic distribution of $\sqrt{k}\left(T_{(m)}-\xi_{\lambda}\right)$ is normal with zero mean and variance $\lambda(1-\lambda) / f^{2}\left(\xi_{\lambda}\right)$. 
As $k \rightarrow \infty, \lambda \rightarrow m / k=c$. From the above result, we see that

$$
E\left[T_{(m)}\right] \rightarrow \xi_{c}=F^{-1}(c)
$$

and

$$
\operatorname{Var}\left[T_{(m)}\right]=\frac{\operatorname{Var}\left[\sqrt{k}\left(T_{(m)}-\xi_{\lambda}\right)\right]}{k} \rightarrow \frac{c(1-c)}{k f^{2}\left(\xi_{c}\right)}
$$

Therefore, we have the following property:

QOS 3. For a given fixed $m / k=c<1$, as $k \rightarrow \infty, E\left[T_{(m)}\right] \rightarrow F^{-1}(c)$ and $\operatorname{Var}\left[T_{(m)}\right] \rightarrow 0$, regardless of the distribution of $T_{i}$.

This basically states that the delay jittcr as defined by $\operatorname{Var}\left[T_{(m)}\right] / E^{2}\left[T_{(m)}\right]$ can be reduced arbitrarily to 0 for a given fixed redundancy factor $1-c$. As with QOS 2, this again points out the suitability of vector routing for traffic with large burst lengths, since the batch size $m$ can then be set to a large value for a constant $c$.

There are several further observations that we can make from QOS 3. First, suppose we require $E\left[T_{(m)}\right]=$ $F^{-1}(c)<T_{\max }$, where $T_{\max }$ is the maximum delay allowed before time out at the receiver end. Since $F[\cdot]$ is a monotonic function, we can write

$$
c<F\left[T_{\max }\right] .
$$

Referring back to Section 3.2, we see that $c<F\left[T_{\max }\right]=p$ if $T_{\min }=0$ (that is, if the buffer size is not a concern at the receiver). Since $\operatorname{Var}\left[T_{(m)}\right] \rightarrow 0$, the result here is consistent with the stable condition obtained in Section 3.2. It is also interesting to note that $\operatorname{Var}\left[T_{(m)}\right] \rightarrow 0$ as long as $c<1$; it is not necessary that $c<p$.

\section{Performance evaluation by simulations}

An ATM network in general consists of a number of ATM switches connected by communication channels (physical links or virtual channels). For simplicity and easy comparison between the parallel and non-parallel schemes, we assume that switch loading is balanced. The source is connected to a switch input and may have many end-to-end connections to different destinations. Each burst of cell arrivals on the input belong to one end-to-end connection and we assume its cells are equally likely to be destined for any $k$ of the $N$ outputs(consider an $N \times N$ switch) because of the traffic splitting mechanism, the outputs of different burst are independent, but cells of the same burst are destined for the same $k$ outputs. This corresponds to the situation where there are many independent virtual connections on the input link, each contributing to a small amount of the total input traffic.

This section investigates the cell-loss probabilities of parallel and non-parallel communications using simulations. Specifically, we focus on the first switch connected to the source only, as shown in Fig. 5. The performance at the first switch is representative of the performance of the other switches except the last switch, as explained below. Assuming balanced distribution of traffic load in the network, an input of an intermediate switch has $k$ times more bursts than an input of the first switch; however, each burst has $k$ times fewer cells and all these cells within the same burst are destined for the same output (note: each 


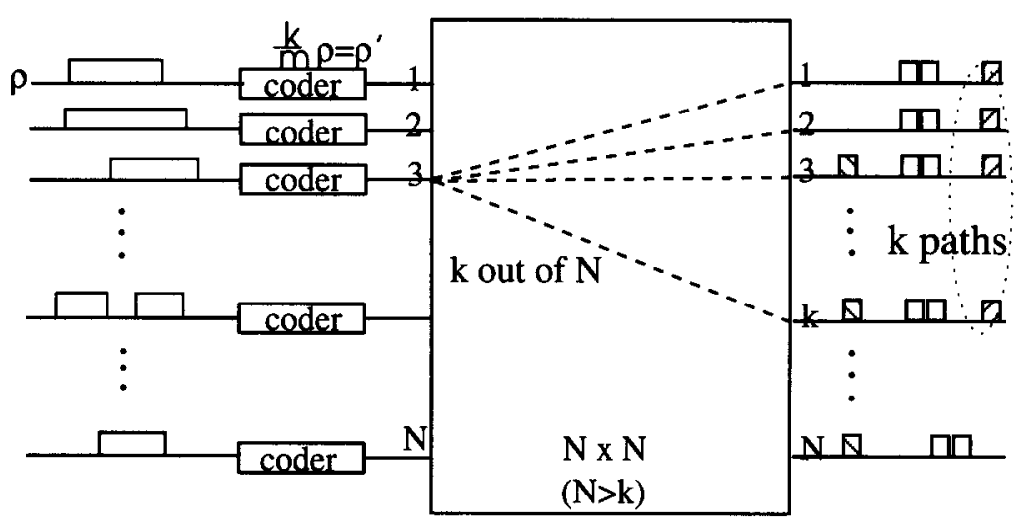

Fig. 5. Parallel paths in first switch.

batch-size(\# of cells)

burst i

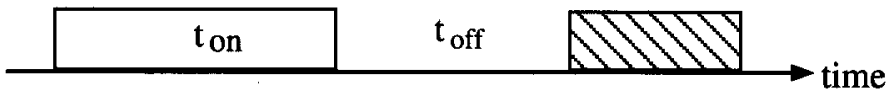

(a) On-off source arriving to input of ATM switch.

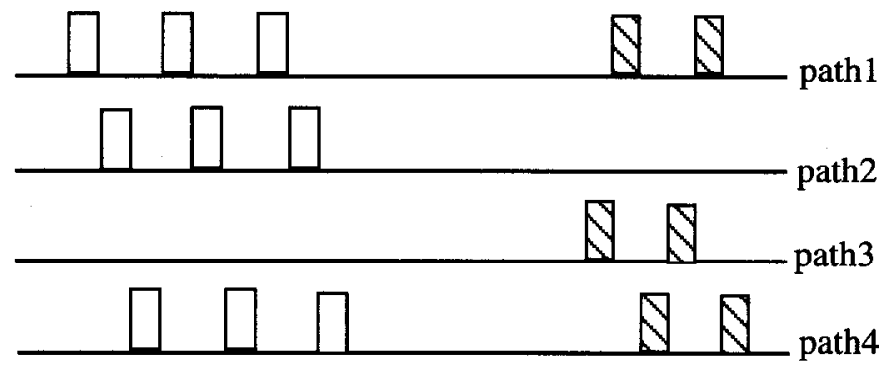

(b) Cells routing in parallel ( 3 out of 4 randomly) for each batch.

Fig. 6. Traffic source model.

burst arriving at an intermediate switch is a sub-burst due to traffic splitting at the first switch). The arrival process at an output is similar to that at an output of the first switch. Therefore, assuming output-buffered switches, the performance at an output should be similar in both cases. By the same token, any performance difference between parallel and non-parallel schemes in the first switch is likely to reflect similar performance difference at the intermediate switches. Unlike at the other switches, the performance of parallel and non-parallel schemes at the last switch for a fixed load is likely to be comparable, especially if the sub-bursts of a burst in the parallel scheme arrives to the last switch at roughly the same time: if the sub-bursts arrive simultaneously, they appear to be like the original large burst as far as their targeted output is concerned.

An "on-off" source model [6,9] is adopted. The source alternates between an on-state, during which cells are transmitted with the source peak bit rate, and an off-state, during which the source is silent. The duration of the two states are exponentially distributed, as shown in Fig. 6. We vary the on-probability and average number of cells during the on-period (i.e. batch size) to obtain several sets of experimental results. 


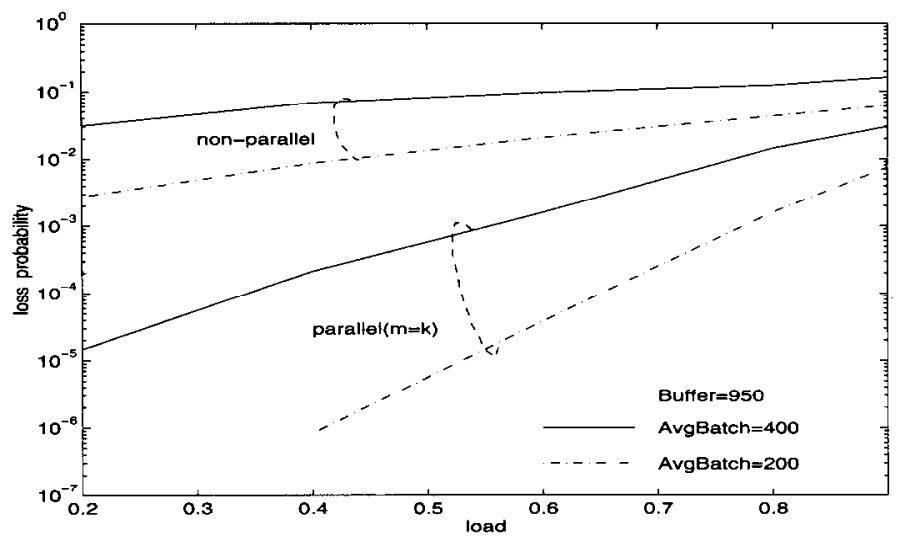

Fig. 7. The loss probability as a functions of offered load.

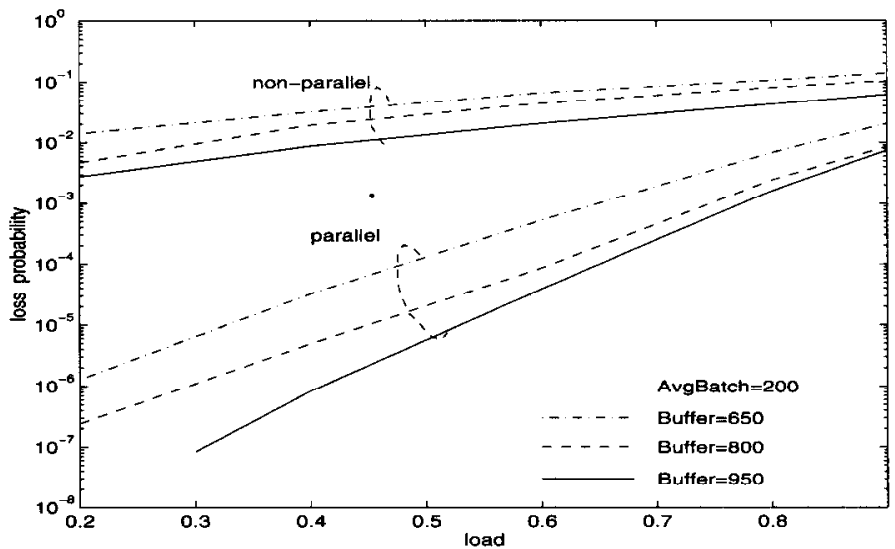

Fig. 8. Comparison of loss probability of parallel and non-parallel with different load and buffer size.

The results of cell-loss probability of each subchannel in a $4 \times 4$ switch are presented in Figs. 7-9. Fig. 7 shows the results of cell-loss probability under different loading conditions for a given buffer size of 950 cells. As can be seen, loss probability of parallel communications with $k=m$ (i.e. splitting without coding) is much smaller than that of non-parallel communications. In addition, for a fixed traffic load, loss probability of parallel commurications is more sensitive to batch size than that of non-parallel communications, i.e. as average batch size reduces, loss probability of parallel communication decreases more quickly. Fig. 8 shows loss probability (for splitting without coding) as a function of loading for different buffer size, fixing average batch size at 200 cells. It is shown that the cell-loss probability of non-parallel communications is much higher than that of parallel communications. Although both the loss probabilities of parallel and non-parallel communications decrease as buffer size increases, loss probability in the latter decrease only slightly when the buffer size increases. When coding is used, the actual traffic load entering the switch increase to $\rho^{\prime}=(k / m) \rho$. Fig. 9 shows the results when the factor $r=k / m$ is varied from 1.0 to 1.3 , with the average batch size and buffer size fixed at 200 cells and 800 cells, respectively. The loss probability of each subchannel is worse than that of $k=m$, since the effective offered load $\rho^{\prime}=(k / m) \rho$ is larger. 


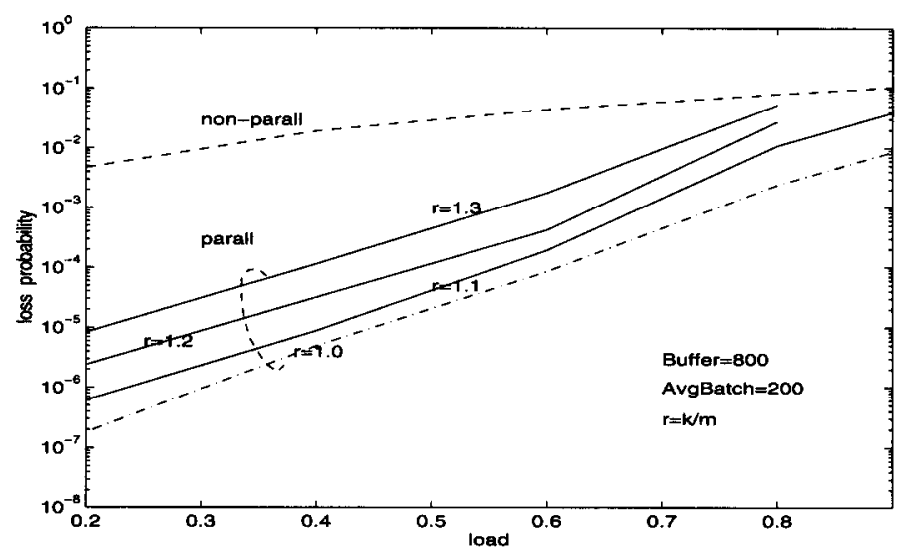

Fig. 9. The loss probability as a function of offered load with different redundancy of coding in parallel scheme.

In general, so long as $(k / m) \rho<0.9$ the probability performance of parallel communication is still better than that of non-parallel communication.

It should be stated that for the parallel scheme, Figs. 7-9 relate to the performance at one of the switches in a virtual subchannel only. The advantage of coding has not been factored in and will become obvious only when the end-to-end performance is considered. For instance, Fig. 9 shows that the loss probability is smallest when the parallel scheme is used without coding $(r=1.0)$. This, however, does not mean that the average mean end-to-end delay will also be smallest in this case: the dispersed information over the $k=m$ subchannels can be considered as received only when information on all $k=m$ subchannels has been received. Coding can be used to decrease the end-to-end mean delay since reception is considered to be complete as soon as only $m<k$ of the $k$ pieces of information arrive at the receiver as discussed in Section 3.3.

Combining Figs. 3 and 9, we can say the following. When the network load is light we can choose large redundancy $\mathrm{k} / \mathrm{m}$ to increase the reliability of information transmission. When the load is heavy, small redundancy factor should be chosen so that the end-to-end performance of parallel communication is still better than that of non-parallel communication.

\section{ATM network control and management}

This section discusses various implications of the VRA for network control and management issues, including the handling of various ATM traffic classes.

\subsection{Application of VRA on ATM traffic classes}

It is important to point out that it is not desirable and necessary to use vector routing for all kinds of services. For instance, for low-bandwidth non-bursty service such as voice, one channel is sufficient. However, there are many situations, especially when traffic is bursty with high peak-rate, where vector routing is appropriate. In the following, we discuss the roles of vector routing in the four classes of services defined for the ATM adaptation layer. 
Class A: Circuit emulation. Circuit emulation traffic from a connection arrives at the source in a continuous fashion. Although it is not bursty in nature, vector routing is still useful when a channel (virtual circuit) with enough capacity for the connection is not available. In this case, we are forced to split the traffic over separate subchannels. An example is when we want to create a $600 \mathrm{Mbit} / \mathrm{s}$ circuit using $150 \mathrm{Mbit} / \mathrm{s}$ physical links. This creates higher delay jitters at the receiver end. For circuit emulation connections, VRA encodes the information in rnultiple of $m$ packets. It waits until enough amount of information is accumulated at the source before the $k$ encoded packets are sent out on separate subchannels.

Class B: Variable bit rate service. For variable bit-rate connections, such as real-time video connections, VRA attempts to encode the information in multiples of $m$ packets. Since the bit- rate is variable, there is a chance that excessive delay may be incurred at the source should the bit rate becomes momentarily very low. We can fix a maximum delay at the source between the arrival of the first bit and the last bit of a batch of packets to be encoded. If information of less than $m$ packet arrives during this period, some dummy packets will be inserted. A consequence of this strategy is that additional bandwidth overhead will be incurred.

Class C: Connection oriented data service. Traffic can be rather bursty for data services. Suppose that packets arrive at the source in bursts that are sparsely separated. Each burst of packets are partitioned into batches of $m$ packets, each with some $g<m$ residual packets. As mentioned in Section 2.2, with the Round-up strategy, empty packets are inserted to make a total of $m$ packets out of these residual packets. With the No-rounding strategy, we momentarily set $m$, hence $k$, to a lower value for the transmission of the residual packets. We can choose an arbitrary subset of the subchannels for the transmission of the residual packets. If the burst length varies widely from burst to burst, the No-rounding strategy is more suitable because of its lower overhead.

Class D: Connectionless data service. An end-to-end setup is not compatible with connectionless services. Nevertheless, we can have a node-to-node setup that employs VRA. A set of $k$ channels can be reserved between two nodes for the exclusive transport of connectionless traffic from different origins. The value of $m$ is chosen to be an appropriate value close to but smaller than $k$. Information must go through the "VRA layer" situated above the ATM layer at each node on way to its destination. As each burst of connectionless traffic enters a node, as discussed for the connection-oriented service, the encoder will attempt to partition it into batches of $m$ packets. Since all the connectionless traffic, regardless of their originating source, will use the same set of $k$ channels, it is likely that $k$ will be chosen to be a large value to accommodate the aggregate traffic. It appears that the No-rounding strategy is more suitable, since the burst length can be widely varying from burst to burst (due to their different origins), and that with a large $m$, it is more likely that burst length itself will be smaller than $m$.

\subsection{Implications for network management}

We now examine the implications of VRA for various network management issues.

Reliability and survivability. By providing redundancy, an end-to-end connection can be made more reliable and survivable. There are two aspects to reliability. Section 2.3 has discussed reliability with regard to packet transmission errors. The other aspect is the network component failures that bring down some of the subchannels. As long as $m$ subchannels survive, the original information can still be reconstructed. It is possible to prove a result similar to QOS 2 that by increasing $k$, a connection can be made as survivable as desired while maintaining a fixed efficiency factor $m / k$. 
Evolution and integration. As the network evolves, more new service types may be introduced. The traffic characteristics of the new service types may be very different from the traffic characteristics of the existing service types. It would be very awkward to have to redesign the network traffic management schemes to accommodate the new service types.

With VRA, a simpler traffic-management philosophy is possible. Only a basic set of subchannel types need be set up in the network. These subchannel types may differ in terms of their carried loads and the allowed traffic burstiness.

As far as traffic management is concerned, the network does not have any concept of different service types. It only knows and manages the subchannels based on their load and burstiness requirements. To introduce a new service, instead of creating a new connection type and modifying the traffic management schemes inside the network, it is mapped on to a number of subchannels of an existing type which the network already knows how to manage. Thus, the network is akin to a general-purpose computer, and mapping services to the subchannels is akin to programming the computer to perform different computation tasks - there is no need to redesign the basic instruction set of the computer each time a new algorithm for certain computation is invented. In this way, VRA facilitates the integration of new services into a network as it evolves.

In an overall ATM network, there are likely to be certain portions of the network with only low-bandwidth channels. For instance, some of the subnetworks could be wireless networks. Parallel channels is a solution for supporting network bandwidth in these subnetworks. Thus, the overall network can be made into a high bandwidth network. VRA again plays a significant role, since merely parallelizing the channels without any encoding may result in poor quality of service for the connection.

Network security. VRA also has some security implications. Namely, it is not possible to reconstruct the original information with less than $m$ packets. An eavedropper who tapped onto a subchannel will not be able decode the information. By having several subchannels, it is difficult for anybody other than the receiver to have access to the complete information. Of course, for higher security, additional encryption is desired.

Administration and billing. One of the problems in billing is how services are to be charged, according to peak-rate usage, number of packets delivered or other schemes. Since a network that adopts VRA has only a fixed number of subchannel types, services can be charged according to the number and types of subchannels they use. This is reasonable since the network treats all subchannels of the same type equally.

\section{Conclusions and future research issues}

This paper has described a parallel communication scheme based on a VRA for end-to-end control in ATM networks. The scheme decomposes a high-bandwidth bursty channel into multiple parallel subchannels. Beneficial effects of VRA include improved load balancing in the network and the smoothing effect on the burstiness of the source traffic.

We have investigated the control of QOS and established the fundamental trade-offs between the redundancy overhead of VRA and QOS. We have argued that VRA is flexible enough to satisfy the QOS of various classes of services, including data and real-time services such as video and image. For any particular connection, we have shown that packet error, loss and delay can be arbitrarily improved with sufficient redundant routes as indicated by QOS1, QOS2 and QOS3.

As for network management and reliability, the parallel communication scheme is highly fault-tolerant and it is robust in the presence of network failure. Security is achieved because the transmitted information is 
scrambled and distributed over several independent subchannels. Thus, it is almost impossible for anyone other than the receiver to access the complete information. Furthermore, the bandwidth requirement of a broadband service can be satisfied by the total bandwidth of a number of subchannels, allowing lowbandwidth networks to carry high-bandwidth services. As a result, the evolution process of the broadband network could be much economical and graceful.

The performance evaluation of parallel communications scheme has also been addressed by simulation. If no coding is used, then $k=m$, and the net effect of the parallel communication scheme is to reduce the traffic burstiness. This improves the delay and loss probability performance at a switch in the path of the virtual connection. If coding is used, then $k>m$, and the network load is large, we need to make sure that the effective load $(\mathrm{k} / \mathrm{m}) \rho$ is smaller than 0.9 in order that the performance is still superior to the non-parallel situation ( $m=k=1$ ) with a load of $\rho$.

The local performance at a switch is the best when parallel communications with no coding ( $m=k$ ) is used; however, the end-to-end performance may not be, considering the fact that the reconstruction of original information based on the information from the subchannels will then be at the mercy of the worstperforming subchaanel. Making $k>m$ will alleviate this problem while retaining the burstiness-reduction advantage of parallel communications. Thus, for a given situation and requirement, there must be a set of optimum $m$ and $k$ for end-to-end performance. This remains to be further investigated.

In conclusion, we have proposed a new parallel communication scheme for application in ATM networks. There are many interesting issues remained to be studied, as described below.

- Burst control and call admission - The main issue here is how to determine the parameters $m$ and $k$ for a call subject to certain burst-control requirements on the network side and service requirements on the user's side. Another key issue is how many subchannel types should be available in order to be able to support a large base of different service types.

- Reconstruction of partial information - In video communication, for example, it is desirable to reconstruct partial images even when some packets are lost. The discussion in this paper has dwelled on the case where either a batch of packets is decoded correctly or incorrectly. An interesting question is whether partial information can be reconstructed if less than $m$ packets are received correctly.

- Wavelength-division-multiplexed (WDM) optical networks - In WDM optical networks, many subchannels can be established using the WDM technique. An interesting issue is how to use the large bandwidth in an optical network to simplify network control and protocol problems. Consider a generic WDM network in which each user is assigned a particular wavelength for transmission. The wavelengths of all users are received by each user. Each receiver then has the responsibility of filtering out the desired wavelength. Generally, complicated protocols are needed for the resolution and determination of who should be talking to whom, at what time, and how a transmitter goes about informing the receiver about its intention to transmit. The VRA framework provides potentially a simple solution. Each receiver is statically tuned to $k$ wavelengths, and each transmitter can transmit simultaneously on $k$ wavelengths, with tunability on each wavelength. To reduce the number of wavelength required in the network and the required tunable range of the transmitter, the same wavelength can be assigned to several receivers, with no receiver having exactly the same $k$ wavelengths overall. Assuming the transmitters do not coordinate with each other, signal collisions may occur on individual wavelengths. Under the VRA framework, however, only $m$ out of $k$ subchannels need to be collision free for correct reception.

- Wireless networks - The problem here is somewhat the reverse of the problem in the WDM network. There are many channels, each with a small capacity. The issue is how to use a number of channels to transport high-bandwidth service. This problem in the context of a circuit-switched network has been addressed. 
In fact, the so-called inverse multiplexers are available commercially to pool a number of small circuit to construct a large circuit. For packet-switched network, synchronized reception of each subchannel becomes even more difficult, since the delay on each subchannel may be changing dynamically over time. In addition, whether a new scheme for channel allocation is required or desirable is not clear.

\section{Appendix A}

This appendix considers the reduction of traffic burstiness using VRA, without the assumption of multiple-m-packets burst length. We shall adopt the notation in Section 2.3. For the source traffic, the number of packets arriving in time slot $i$ is

$$
y[i]=m f[i]+g[i] .
$$

Consider a particular subchannel $p$. The $m f[i]$ packets from the source traffic will generate $f[i]$ packets on this subchannel. The $g[i]$ residual packets will generate one or no packet based on the "No-rounding" strategy (see Section 2.3). Thus, the number of packets arriving at subchannel $p$ is

$$
y_{p}[i]=f[i]+b_{p}[i],
$$

where $b_{p}[i]$ is a random variable depending on $g[i]$. We shall assume that $k^{\prime} \approx g[i] k / m$ of the subchannels will be chosen at random to carry the residual packets. Thus,

$$
\operatorname{Pr}\left[b_{p}[i]=1\right] \approx \frac{g[i]}{m}, \quad \operatorname{Pr}\left[b_{p}[i]=0\right] \approx 1-\frac{g[i]}{m} .
$$

For simplicity, we shall assume strict equality of the above relationships in the following. We shall denote the expectation of a random variable, say $x$, given $g[k] \forall k$ by $E[x \mid g[k]]$. We have

$$
\begin{aligned}
E[b[i] \mid g[k]] & =\frac{g[i]}{m}, \\
E\left[b^{2}[i] \mid g[k]\right] & =\frac{g[i]}{m}, \\
E[b[i] b[j] \mid g[k]] & =\frac{g[i] g[j]}{m^{2}}, \quad i \neq j .
\end{aligned}
$$

Now, consider the averaged number of arrivals within a time window of $T, Y_{p}[i]$ (see Section 2.3). Let us denote expectation taken over all possible $g[i]$ as $E_{g[i]}[\cdot]$. We have

$$
\begin{aligned}
E\left[Y_{p}^{2}[i]\right]= & \frac{1}{T^{2}} E_{g[i]}\left[E\left[Y_{p}^{2}[i] \mid g[k]\right]\right] \\
= & \frac{1}{T^{2}} E_{g[i]}\left[E\left[\sum_{i-T<t, u \leq i}\left(f[t]+b_{p}[t]\right)\left(f[u]+b_{p}[u]\right) \mid g[k]\right]\right] \\
= & \frac{1}{T^{2}} E_{g[i]}\left[\sum_{i-T<t, u \leq i} E[f[t] f[u] \mid g[k]]+E\left[b_{p}[t] \mid g[k]\right] E[f[u] \mid g[k]]\right. \\
& +E\left[b_{p}[u] \mid g[k]\right] E[f[t] \mid g[k]]+E\left[b_{p}[t] b_{p}[u] \mid g[k]\right]
\end{aligned}
$$




$$
\begin{aligned}
= & \frac{1}{T^{2}} \sum_{\substack{i \neq u \\
i-T<t, u \leq i}} E[f[t] f[u]]+\frac{E[f[u] g[t]]}{m}+\frac{E[f[t] g[u]]}{m}+\frac{E[g[t] g[u]]}{m^{2}} \\
& +\frac{1}{T^{2}} \sum_{i-T<t \leq i} E\left[f^{2}[t]\right]+\frac{2 E[f[t] g[t]]}{m}+\frac{E[g[t]]}{m}
\end{aligned}
$$

Notice the subtlety in the third line above that even if $b_{p}[\cdot]$ and $f[\cdot]$ were dependent, they are independent given $g[\cdot]$, since $b_{p}[\cdot]$ is completely determined by $g[\cdot]$. Now, for the source traffic

$$
E\left[Y^{2}[i]\right]=E\left[N^{2}[i]\right]+\rho^{2}=\frac{1}{T^{2}} E\left[\sum_{i-T<t, u \leq i}(m f[t]+g[t])(m f[u]+g[u])\right] .
$$

Comparing (A.10) and (A.11), we have

$$
E\left[Y_{p}^{2}[i]\right]=\frac{E\left[N^{2}[i]\right]}{m^{2}}+\frac{\rho^{2}}{m^{2}}+\frac{1}{T}\left[\frac{E[g[i]]}{m}-\frac{E\left[g^{2}[i]\right]}{m^{2}}\right],
$$

where we have assumed the stationarity of $g[i]$. Therefore, the traffic burstiness on subchannel $p$ is

$$
\begin{aligned}
B_{p}[i] & =E\left[N_{p}^{2}[i]\right]=E\left[Y_{p}^{2}[i]\right]-\frac{\rho^{2}}{m^{2}} \\
& =\frac{B[i]}{m^{2}}+\frac{1}{T}\left[\frac{E[g[i]]}{m}-\frac{E\left[g^{2}[i]\right]}{m^{2}}\right] .
\end{aligned}
$$

As in Section 2.3, if we consider the multiplexing of decomposed traffic from $m$ identical sources onto the same channel, the burstiness of the composite traffic is

$$
B_{s}[i]=\frac{B[i]}{m}+\frac{1}{T}\left[E[g[i]]-\frac{E\left[g^{2}[i]\right]}{m}\right] \text {. }
$$

\section{References}

[1] E.W. Biersack, A simulation study of forward error correction in ATM networks, Computer Comm. Rev., SIGCOMM 22 (1) (1992) $36-47$.

[2] K.L. Chung, Elementary Probahility Theory with Stochastic Processes, Springer, Berlin (1974).

[3] J.H. Dejean, L. Dittmann and C.N. Lorenzen, String mode - A new concept for performance improvement of ATM networks, IEEE J. Selected Areas Comm. 9 (9) (1991).

[4] S. Kotz and N.L. Johnson, Encyclopedia of Statistical Sciences, Vol. 6, Wiley, New York (1985).

[5] T.T. Lee and S.C. Liew, Broadband packet switches based on dilated interconnected networks, IEEE Trans. Comm., to appear.

[6] S.C. Liew and T.T. Lee, A fundamental property governing traffic management in ATM network, Conf. Record., IEEE Infocom'93 (1993) 1240-1250.

[7] N.F. Maxemchuk, Dispersity routing in store-and forward networks, Ph.D. Dissertation, University of Pennsylvania.

[8] A.J. McAuley, Reliable broadband communications using a burst erasure correcting code, Proc. ACM SIGCOMM 90 (1990) 287-306.

[9] M.G. Hluchyj, M.J. Karol, Queueing in high-performance packet switching, IEEE J. Select. Areas Comm. 6 (9) (1988) 1587-1597. 
[10] H. Nyquist, Certain topics in telegraph transmission theory, Trans. AIEE 47 (1928) 617-648.

[11] M.O. Rabin, Efficient dispersal of information for security, load balancing and fault tolerance, J. Assoc. Comput. Mach. 36 (2) (1989) 335-348.

[12] I.S. Reed and G. Soloman, Polynomial codes over certain finite fields, J. Soc. Ind. Appl. Math. 8 (1960) 300-301.

[13] V.K. Rohatgi, An Introduction to Prabability Theory and Mathematical Statistics, Wiley, New York (1976).

[14] S. Ross, A First Course in Probability, 2nd ed., Macmillan, New York (1984).

[15] A. Weiss, An introduction to large deviations for communication networks, IEEE J. Select. Areas Comm. 13 (6) (1995) 938-952.

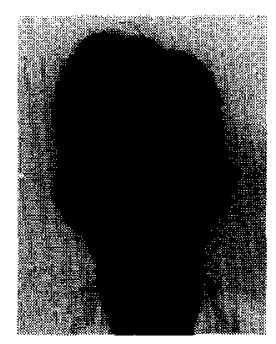

Tony T. Lee received his BSEE degree from National Cheng Kung University Taiwan in 1971, and his MS and Ph.D. degrees in electrical engineering from Polytechnic University in New York in 1976 and 1977, respectively. Currently, he is a Professor of Information Engineering at the Chinese University of Hong Kong, and an adjunct Professor of the Institute of Applied Mathematics of Chinese Academy of Science. From 1991 to 1993, he was a Professor of Electrical Engineering at Polytechnic University of New York, Brooklyn, New York. He was with AT\&T Bell Laboratories, Holmdel, NJ, from 1977 to 1983, and Bellcore, Morristown, NJ, from 1983 to 1993 . He was an adjunct faculty member in the Electrical Engineering Department of Columbia University for the Fall term of the 1989 academic year. He also taught a pilot course on fast packet switches at MIT in 1990 . He was selected to be a Distinguished Member of Professional Staff by Bellcore since 1988. He is the recipient of the 1988 Leonard G. Abraham prize paper award from the IEEE Communications Society.

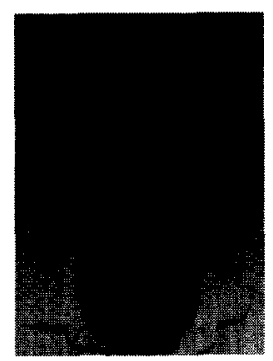

Soung C. Liew received his S.B., S.M., and Ph.D. from M.I.T. He joined Bellcore in New Jersey, USA, in March 1988. Since July 1993, he has been with Department of Information Engineering, The Chinese University of Hong Kong. He is active in various research areas related to broadband communications, including fast packet switching, broadband network control, video transport, and optical networking. His recent research efforts focus on the processing and adaptation of video information for efficient network transport. He initiated and is coordinating the first ATM testbed network which interconnects three major universities in Hong Kong. An ongoing prototyping project is to build a Wedlike multimedia delivery and presentation system to explore how advanced multimedia and networking technologies can be used to improve the learning/teaching process.

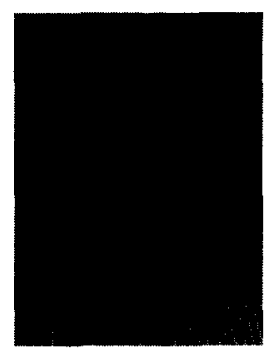

Qua-Long Ding received the B. Eng. and M. Eng. degrees in electrical engineering from Xidian University, Xi'an, China, in 1982 and 1987, respectively. From 1982 to 1993 he was a lecturer in the Department of Information Engineering of Xidian University and a research member in the National Key Laboratory of the Theory and Key Technology of ISDN, where he engaged in the design and implement integrated service of LAN and wireless LAN. From 1993, he is a Ph.D. candidate and research assistant in the Department of Information Engineering, The Chinese University of Hong Kong. His current research interests include broadband network traffic management and flow control design of ATM switch, parallel communications in ATM networks and wireless LANs. 